\title{
Hopf Bifurcation from Relative Periodic Solutions; Secondary Bifurcations from Meandering Spirals
}

\author{
Jeroen S. W. Lamb * Ian Melbourne ${ }^{\dagger} \quad$ Claudia Wulff ${ }^{\dagger}$ \\ 21 February, 2006; Updated 30 May 2006

\begin{abstract}
Dedicated to André Vanderbauwhede on the occasion of his 60th birthday
\end{abstract}

\begin{abstract}
We consider nonresonant and weakly resonant Hopf bifurcation from periodic solutions and relative periodic solutions in dynamical systems with symmetry. In particular, we analyse phase-locking and irrational torus flows on the bifurcating relative tori.

Results are obtained for systems with compact and noncompact symmetry group. In the noncompact case, we distinguish between bounded and unbounded dynamics. Applications of our results include secondary Hopf bifurcation from meandering multiarmed spirals.
\end{abstract}

\section{Introduction}

In recent work $[17,18,24]$, we have developed a systematic theory for the study of local bifurcations from periodic solutions and relative periodic solutions in dynamical systems with symmetry. The theory proceeds by relating the problem to a simpler problem studied previously [12]; namely local bifurcation from a fully symmetric equilibrium.

The aim of the paper is twofold. On the one hand, we discuss an important example - spiral wave dynamics in excitable media, building upon previous work of Sandstede et al. [23] in the case of one-armed spirals, and extending the results to multi-armed spirals. This can be seen as both an illustration and an application of the methods in [17, 18, 24]. On the other hand, the treatment of this example requires several new techniques, specific to Hopf bifurcation from relative periodic solutions in systems with symmetry, to understand the effects of phaselocking, resonance tongues, and quasiperiodic flows. We consider these

\footnotetext{
*Department of Mathematics, Imperial College London, London SW7 2AZ, UK

†Department of Mathematics \& Statistics, University of Surrey, Guildford, Surrey GU2 7XH, UK
} 
issues for both compact symmetry groups and certain noncompact symmetry groups such as the Euclidean group.

Suppose that $\Gamma$ is a compact Lie group acting orthogonally on $\mathbb{R}^{n}$ and $F: \mathbb{R}^{n} \rightarrow \mathbb{R}^{n}$ is a smooth vector field satisfying the $\Gamma$-equivariance condition $F(\gamma x)=\gamma F(x)$ for $\gamma \in \Gamma$ and $x \in \mathbb{R}^{n}$. A solution $x(t)$ for the corresponding flow is called a relative equilibrium if $x(t) \in \Gamma x(0)$ for all $t \in \mathbb{R}$. A solution $x(t)$ is called a relative periodic solution if $x(t)$ is not a relative equilibrium but there exists a $T>0$ such that $x(T) \in \Gamma x(0)$. The least such number $T$ is called the relative period.

Let $P=\{x(t)\}$ be a relative periodic solution. Define

$$
\Delta=\{\gamma \in \Gamma: \gamma x(0)=x(0)\}, \quad \Sigma=\{\gamma \in \Gamma: \gamma \bar{P}=\bar{P}\}
$$

We call $\Delta$ the spatial symmetry group and $\Sigma$ the spatiotemporal symmetry group. These are closed subgroups of $\Gamma$ and $\Delta$ is a normal subgroup of $\Sigma$. We say that the (relative) periodic solution is $(\Delta, \Sigma)$-symmetric.

An alternative definition of $\Sigma$ is that it is the closed subgroup of $\Gamma$ generated by $\Delta$ and $\sigma \in \Gamma$, where $x(T)=\sigma x(0)$ and $T$ is the relative period. Such an element $\sigma$ is called a spatiotemporal generator.

If $\operatorname{dim} \Delta=\operatorname{dim} \Gamma$, then the dynamics in a vicinity of the periodic solution depends only on $\Sigma$, so we may suppose without loss that $\Gamma=\Sigma$ in such situations. Hence, there is the following hierarchy of situations of increasing complexity in which to consider Hopf bifurcation:

(0) $\Delta_{0}$-symmetric equilibrium for a $\Delta_{0}$-equivariant vector field.

(1) $(\Delta, \Delta)$-symmetric periodic solution (purely spatial symmetry) for a $\Delta$-equivariant vector field.

(2) $(\Delta, \Sigma)$-symmetric periodic solution (spatial and spatiotemporal symmetry) for a $\Sigma$ equivariant vector field where $\Sigma / \Delta \cong \mathbb{Z}_{m}$ with $m \geq 2$.

(3) $(\Delta, \Sigma)$-symmetric relative periodic solution for a $\Gamma$-equivariant vector field where $\operatorname{dim} \Gamma>\operatorname{dim} \Delta$.

There is a well-developed theory for case (0): Hopf bifurcation from a fully symmetric equilibrium [12]. The aim of this paper is to show how all the remaining cases reduce to case (0) so that bifurcation-theoretic phenomena in cases (1)-(3) can to a large extent be "read off" from the known results for case (0).

Remark 1.1 In reducing from case (1) to case (0), it turns out that $\Delta_{0}=\Delta$. However, in reducing from cases (2) and (3), $\Delta_{0}$ is a cyclic extension of $\Delta$.

We focus on three main issues: branching patterns of (relative) invariant tori, phaselocking, and conjugation to a linear torus flow. In case (3), we allow that $\Gamma$ is not necessarily compact (it is required that $\Delta$ is compact). Moreover, in the case of Euclidean symmetry 
in the plane, $\Gamma=\mathbf{S E}(2)$, we consider the question of compactness of solutions on relative invariant tori. This is especially important for the application to spirals, where $\Delta=\mathbb{Z}_{\ell}$ ( $\ell=1$ for one-armed spirals and $\ell \geq 2$ for multi-armed spirals).

It turns out that such issues have been only partially studied even in case (1). Hence Sections 2 and 3 are devoted to understanding this "simplest case". Section 2 contains some basic facts about equivariant circle diffeomorphisms, such as rotation numbers and the equivariant Denjoy theorem. In Section 3, we consider the reduction of case (1) to case (0). In the process, we recover and substantially extend results of Chossat \& Golubitsky [6] on Hopf bifurcation from a periodic solution with purely spatial symmetry.

In Section 4, we turn to Hopf bifurcation from periodic solutions with spatial and spatiotemporal symmetry (also known as discrete rotating waves). Here, we apply the ideas in $[17,18]$ to reduce from case $(2)$ to case $(0)$, but elaborating on the issues of phaselocking and linearisation.

In Section 5, we briefly consider Hopf bifurcation from general relative periodic solutions with compact spatial symmetry $\Delta$. Finally, in Section 6 , we consider the application to bifurcations from one-armed and multi-armed spirals. Here, $\Gamma=\mathbf{S E}(2)$ is noncompact, and we address also the issue of determining whether the dynamics on the bifurcating phaselocked relative periodic solutions and relative invariant tori is bounded or unbounded.

\section{Equivariant circle diffeomorphisms}

In this section, we collect together some elementary results about equivariant circle diffeomorphisms. We do not claim that these results are new, but explicit statements are hard to find in the literature. (In particular, we have not seen elsewhere the equivariant Denjoy theorem, Proposition 2.3 and Corollary 2.4 below.) A general reference for the nonequivariant statements below is [13, Section 6.2].

Let $C$ be an oriented circle, and suppose that $f: C \rightarrow C$ is an orientation preserving homeomorphism. We assume that $f$ is $\mathbb{Z}_{r}$-equivariant with respect to a free action of $\mathbb{Z}_{r}$ on $C$. As usual, we define the rotation number $\rho(f)=\lim _{n \rightarrow \infty} F^{n}\left(t_{0}\right) / n$ where $F: \mathbb{R} \rightarrow \mathbb{R}$ is a lift of $f$, and $\rho(f)$ is independent of choice of $F$ and $t_{0}$.

Suppose that $f$ has rotation number $p / q$ (written in its lowest terms). Then (i) there exists a periodic orbit for $f$, (ii) all periodic orbits on $C$ have period $q$, and (iii) all other trajectories on $C$ are asymptotic to the periodic orbits. Generically, the periodic orbits are hyperbolic and hence the asymptotic dynamics consist of a finite number of periodic sink/source pairs of period $q$.

Our first result is implicit in a more general result of Krupa \& Roberts [16, Lemma 3.8] on (noninvertible) equivariant circle maps.

Proposition 2.1 Suppose that $\mathbb{Z}_{r}$ acts freely on the circle $C$ and that $f: C \rightarrow C$ is a $\mathbb{Z}_{r^{-}}$ equivariant homeomorphism with rotation number $p / q$. Then the periodic orbits on $C$ have spatiotemporal symmetry $\mathbb{Z}_{s}$ where $s=\operatorname{gcd}(q, r)$. 
Proof Let $\widetilde{C}=C / \mathbb{Z}_{r}$. An easy argument [16, Lemma 3.1] shows that the quotient map $\tilde{f}: \widetilde{C} \rightarrow \widetilde{C}$ has rotation number $r p / q$. This reduces to $r^{\prime} p / q^{\prime}$ in its lowest terms, where $r^{\prime}=r / s, q^{\prime}=q / s$.

Let $x \in C$ be a periodic point for $f$, so $x$ has period $q$. At the quotient level, $x$ has period $q^{\prime}$ for $\tilde{f}$. Hence $f^{q}(x)=x$ and $f^{q^{\prime}}(x) \in \mathbb{Z}_{r} \cdot x$, with $q, q^{\prime}$ least. It follows that the corresponding periodic orbit has symmetry $\mathbb{Z}_{q / q^{\prime}}=\mathbb{Z}_{s}$ as required.

In the obvious terminology, $p / q$-phaselocked periodic points on $C$ have absolute period $q$ and relative period $q / s$.

This result can be strengthened slightly. If $y \in C$, then the group orbit $\mathbb{Z}_{r} \cdot y \subset C$ has an ordering $y=y_{1}<y_{2}<\cdots<y_{r-1}$ with respect to the orientation on $C$. We distinguish the element $\sigma \in \mathbb{Z}_{r}$ such that $y_{2}=\sigma y_{1}$. (It follows that $\sigma y_{j}=y_{j+1}$ where $j+1$ is computed $\bmod r$.) This definition of $\sigma$ does not depend on $y$. If $C$ is the unit circle in $\mathbb{R}^{2} \cong \mathbb{C}$ and $\mathbb{Z}_{r}$ acts linearly, then $\sigma$ is the element of $\mathbb{Z}_{r}$ that acts as $z \mapsto e^{2 \pi i / r} z$.

We already have that $f^{q / s}(x) \in \mathbb{Z}_{r} \cdot x$ for all periodic points $x \in C$ but this is strengthened to:

Proposition 2.2 Under the hypotheses of Proposition 2.1, $f^{q / s}(x)=\sigma^{p r / s} x$ for all $x \in C$ periodic.

Proof Observe that $\rho\left(f^{q / s}\right)=(q / s) \rho(f)=p / s$. The periodic point $x$ has period $s$ for $f^{q / s}$ and consists of a single $\mathbb{Z}_{s}$ group orbit. Hence $f^{q / s}(x)=\sigma^{d} x$ for some $d \geq 1$. After $s$ iterates, we obtain $f^{q}(x)=\sigma^{s d} x=x$. Since the rotation number is $p / s$, this corresponds to winding $p$ times around the circle, so $s d / r=p$. Hence, $d=p r / s$ as required.

In the terminology of the introduction, Propositions 2.1 and 2.2 state that the periodic points on $C$ are $\left(1, \mathbb{Z}_{s}\right)$-symmetric with spatiotemporal generator $\sigma^{\mathrm{pr} / \mathrm{s}}$.

Next, we consider the case when the rotation number $\rho(f)=\omega$ is irrational. If $f$ is sufficiently smooth ( $C^{2}$ suffices) then by Denjoy's Theorem, $f$ is topologically conjugate to the rigid irrational rotation $x \mapsto x+\omega$. We prove an equivariant version of Denjoy's Theorem.

Proposition 2.3 Let $f: C \rightarrow C$ be a $\mathbb{Z}_{r}$-equivariant homeomorphism of the circle with irrational rotation number. Suppose that $\phi: C \rightarrow C$ is a topological conjugacy between $f$ and a rigid irrational rotation. Then $\phi$ is $\mathbb{Z}_{r}$-equivariant.

Proof Let $\omega$ be the rotation number of $f$. It is well-known (see for example [13, p. 302]) that the conjugacy $\phi$ from $f$ to $R_{\omega}$ is unique up to a rigid rotation. Set $\widetilde{\phi}=R_{2 \pi / r}^{-1} \phi R_{2 \pi / r}$. By $\mathbb{Z}_{r}$-equivariance of $f$,

$$
\widetilde{\phi} f=R_{2 \pi / r}^{-1} \phi R_{2 \pi / r} f=R_{2 \pi / r}^{-1} \phi f R_{2 \pi / r}=R_{2 \pi / r}^{-1} R_{\omega} \phi R_{2 \pi / r}=R_{\omega} \widetilde{\phi}
$$

Hence, $\widetilde{\phi}$ is also a topological conjugacy and so it follows from uniqueness that $\widetilde{\phi} \phi^{-1}=R_{\beta}$ for some $\beta \in[0,2 \pi)$. Moreover,

$$
\phi R_{2 \pi / r} \phi^{-1}=R_{2 \pi / r}\left(R_{2 \pi / r}^{-1} \phi R_{2 \pi / r}\right) \phi^{-1}=R_{2 \pi / r} \widetilde{\phi} \phi^{-1}=R_{2 \pi / r} R_{\beta}=R_{2 \pi / r+\beta} .
$$


This means that $\phi$ defines a topological conjugacy between the rigid rotations with rotation numbers $2 \pi / r$ and $2 \pi / r+\beta$. Since rotation number is an invariant of topological conjugacy, it follows that $\beta=0$ and so $\phi=\widetilde{\phi}=R_{2 \pi / r}^{-1} \phi R_{2 \pi / r}$. Hence $\phi$ is $\mathbb{Z}_{r}$-equivariant.

Corollary 2.4 Suppose that $f: C \rightarrow C$ is a $\mathbb{Z}_{r}$-equivariant $C^{2}$-diffeomorphism with irrational rotation number. Then there exists a $\mathbb{Z}_{r}$-equivariant homeomorphism $\phi: C \rightarrow C$ that topological conjugates $f$ to a rigid irrational rotation.

Proof This is immediate from Denjoy's Theorem and Proposition 2.3.

If the rotation number is Diophantine, as is the case for a set of irrational numbers of full measure, and $f$ is smooth enough, then Arnold's Theorem [1] implies that $f$ is smoothly conjugate to a rigid rotation. By Proposition 2.3, this smooth conjugacy is $\mathbb{Z}_{r}$-equivariant.

The following result is required in Section 4.

Proposition 2.5 Let $\Delta=\mathbb{Z}_{r}$ be a finite cyclic group acting freely on the circle $C$ and let $\phi \in \operatorname{Aut}(\Delta)$ be an automorphism. Let $f: C \rightarrow C$ be an orientation preserving circle diffeomorphism and suppose that $f$ is twisted equivariant: $f \delta=\phi(\delta) f$ for all $\delta \in \Delta \subset S^{1}$. Then $\phi$ is the trivial automorphism (and so $f$ is $\Delta$-equivariant).

Proof The condition for twisted equivariance can be rewritten as $\phi(\delta)=f \delta f^{-1}$. Thus $\phi(\delta)$ is topologically conjugate to $\delta$ by an orientation-preserving conjugacy (namely $f$ ) and hence $\phi(\delta)$ has the same rotation number as $\delta$. But $\Delta$ acts freely and so distinct elements of $\Delta$ have distinct rotation numbers. Hence $\phi(\delta)=\delta$.

\section{$3 \quad$ Hopf bifurcation from periodic solutions with purely spatial symmetry}

In this section, we describe how Hopf bifurcation from a $(\Delta, \Delta)$-symmetric periodic solution $P$ for a $\Delta$-equivariant flow reduces to Hopf bifurcation from a $\Delta_{0}$-symmetric equilibrium (in the notation from the introduction). Since $\Delta=\Delta_{0}$, we write $\Delta$ throughout this section.

Let $X$ be a $\Delta$-invariant local cross-section intersecting $P$ in a single point $x_{0}$. Relabel $x_{0}=0$. The Poincaré map $f: X \rightarrow X$ is $\Delta$-equivariant, and $f(0)=0$. Hopf bifurcation from $P$ for the underlying flow is equivalent to Hopf bifurcation from the fixed point 0 for the Poincaré map $f$.

In Subsection 3.1, we recall results of [6] on Hopf bifurcation for equivariant diffeomorphisms. These results are substantially improved upon in Subsection 3.2 using the approximation $f \sim \exp h$ where $\exp h$ is the time-one map for a $\Delta$-equivariant vector field $h$. In Subsection 3.3, we relate symmetry properties of periodic solutions for $h$ to those of invariant circles of $f$. In Subsection 3.4, we consider the dynamics of $f$ on an invariant circle (phase-locking/irrational rotation). In Subsection 3.5, we relate these issues back to the Hopf bifurcation from the periodic solution $P$. 


\subsection{Hopf bifurcation for equivariant diffeomorphisms}

Introducing a bifurcation parameter $\lambda \in \mathbb{R}$, we suppose that the $\Delta$-equivariant diffeomorphism $f: X \times \mathbb{R} \rightarrow X$ satisfies $f(0, \lambda) \equiv 0$ and that $(d f)_{0,0}$ has complex conjugate eigenvalues on the unit circle (so there is a Hopf bifurcation at $\lambda=0$ ). In particular, we suppose that $(d f)_{0,0}$ has eigenvalues $e^{ \pm 2 \pi i \omega}$ where $\omega \in\left(0, \frac{1}{2}\right)$. Generically, $(d f)_{0,0}$ is semisimple and there are no further eigenvalues on the unit circle, but the eigenvalues $e^{ \pm 2 \pi i \omega}$ may be multiple due to symmetry. After centre manifold reduction, we may suppose without loss that $(d f)_{0,0}$ is semisimple with spectrum consisting entirely of the (multiple) eigenvalues $e^{ \pm 2 \pi i \omega}$.

Write $(d f)_{0,0}=e^{A}$ where $A$ has purely imaginary eigenvalues $\pm 2 \pi i \omega$. Then $e^{t A}$ generates a free action of $S^{1}$ on $\mathbb{R}^{n}$. Altogether, there is an action of $\Delta \times S^{1}$ on $\mathbb{R}^{n}$.

Chossat \& Golubitsky [6] proved the existence of certain classes of invariant circles for the diffeomorphism $f$. Let $J \subset \Delta \times S^{1}$ be an isotropy subgroup with fixed-point subspace Fix $J \subset \mathbb{R}^{n}$. The isotropy subgroup is $\mathbb{C}$-axial if $\operatorname{dim}$ Fix $J=2$.

Theorem 3.1 ( [6, Theorem 3.1] ) If $J$ is a $\mathbb{C}$-axial isotropy subgroup of $\Delta \times S^{1}$, then generically there exists a unique branch of circles $C_{\lambda} \subset \mathbb{R}^{n}$ bifurcating from 0 such that

(i) $f(x, \lambda) \in C_{\lambda}$ for all $x \in C_{\lambda}$,

(ii) $\delta C_{\lambda}=C_{\lambda}$ for all $(\delta, \theta) \in J$, and

(iii) The branch $C_{\lambda}$ is tangent to $\operatorname{Fix} J$ at 0 .

Proof (Sketch) First suppose that $f$ is $\Delta \times S^{1}$-equivariant. Then Fix $J$ is invariant under $f$ and the result follows from the standard Hopf Theorem (see for example [21]) for maps

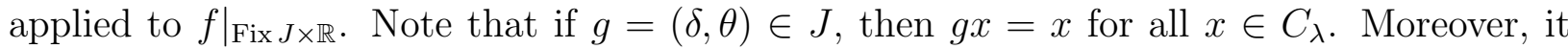
is easy to verify that Fix $J$ is invariant under $S^{1}$ so in particular $\theta C_{\lambda}=C_{\lambda}$ for all $\theta \in S^{1}$. Hence $\delta C_{\lambda}=C_{\lambda}$.

If $\omega$ is irrational, then by Birkhoff normal form theory, $f$ may be assumed to be $\Delta \times$ $S^{1}$-equivariant through arbitrarily high (but finite) order. Moreover to obtain $\Delta \times S^{1}$ equivariance through any specified finite order, it suffices to exclude finitely many rational values of $\omega$. Hence, it remains to show that the invariant circles persist under high enough order $S^{1}$-symmetry breaking perturbations.

When $\operatorname{Fix}(J \cap \Delta)$ is two-dimensional, such perturbation results were addressed in $\mathrm{Ru}-$ elle [21]. In general, we require finite determinacy results of Field [8]. First, there exists an integer $d$ such that the branch $C_{\lambda}$ consists of normally hyperbolic invariant circles provided that the Taylor coefficients of $f$ through order $d$ satisfy finitely many polynomial inequalities. Moreover, there exists a larger integer $D$ such that the branch $C_{\lambda}$ persists if there are higher order terms that break the $S^{1}$ symmetry provided $\Delta \times S^{1}$-equivariance is maintained through order $D$. In general, such a perturbation will destroy $S^{1}$-invariance of $C_{\lambda}$, but by uniqueness $\delta$-invariance is preserved for all $(\delta, \theta) \in J$. 
Remark 3.2 If $\omega$ is irrational, then the Hopf bifurcation is called nonresonant. If $\omega$ is rational, the bifurcation is resonant. As seen in the last proof, for certain purposes it is sufficient to exclude $\omega=p / q$ for finitely many choices of $p$ and $q$. Such bifurcations are weakly resonant and the remaining finitely many cases are called strongly resonant.

\subsection{An alternative proof of Theorem 3.1}

An alternative approach is to approximate the $\Delta$-equivariant diffeomorphism $f$ by the timeone map of a vector field (see for example [18]). Suppose first that $\omega$ is irrational. Then through arbitrarily high order $f=\exp h$ is the time-one map of a $\Delta$-equivariant vector field $h$ with $h(0, \lambda) \equiv 0$ and $(d h)_{0,0}=2 \pi i \omega$. Transforming $f$ into Birkhoff normal form, we may suppose that $h$ is $\Delta \times S^{1}$-equivariant. The equivariant Hopf theorem [11] guarantees the existence of periodic solutions corresponding to any $\mathbb{C}$-axial isotropy subgroup $J \subset \Delta \times S^{1}$.

When $\omega=p / q$ is rational, through arbitrarily high order $f=e^{2 \pi i p / q} \exp h=\exp h e^{2 \pi i / q}$ where $h$ is a $\Delta \times \mathbb{Z}_{q}$-equivariant vector field satisfying $h(0, \lambda) \equiv 0$ and $(d h)_{0,0}=0$. As $q$ increases, $h$ is $S^{1}$-equivariant through higher and higher order. For sufficiently large $q$ (the weakly resonant case) it follows from Field \& Richardson [9] that the equivariant Hopf theorem still yields periodic solutions for any $\mathbb{C}$-axial isotropy subgroup $J$.

Remark 3.3 In the analysis of Hopf bifurcation from a fully symmetric equilibrium with symmetry $\Delta$ (case $(0)$ in the introduction), passing to Birkhoff normal form leads to an $S^{1}$ symmetry to arbitrarily high order. Hence both case (0) and case (1) with $\omega$ irrational are governed to arbitrarily high order by a $\Delta \times S^{1}$-equivariant vector field $h$, achieving our aim of reducing from case (1) to case (0). For weakly resonant $\omega$, case (1) is governed to "sufficiently high order" by such a vector field $h$.

A major advantage of this approach is that the entire bifurcation analysis for the diffeomorphism $f$ is identical (subject to one caveat) to the bifurcation analysis for the vector field $h$. The caveat is that $\exp h$ approximates $f$ only through finite order. Certain aspects of the dynamics, such as phaselocking, may depend delicately on terms in the tail.

Fiedler's theorem and branching patterns For Hopf bifurcation of equivariant vector fields $h$, it is often possible to prove existence of periodic solutions other than those guaranteed by the equivariant Hopf theorem. For example, Fiedler's Theorem [7] guarantees that $h$ has branches of periodic solutions with isotropy $J \subset \Delta \times S^{1}$ for any maximal isotropy subgroup $J$ (regardless of the dimension of Fix $J$ ). The determinacy theorem of Field \& Richardson [9] applies equally well to all isotropy subgroups ( $\mathbb{C}$-axial and non- $\mathbb{C}$ axial, maximal and nonmaximal). Hence, generically such periodic solutions correspond also to invariant circles for the diffeomorphism $f$. The determinacy theory also applies to relative periodic solutions for $h$ and hence to relative invariant circles for the diffeomorphism $f$.

Field \& Richardson [9] speak of branching patterns for equivariant bifurcations. Adopting their terminology, we speak of branching patterns for $\Delta$-equivariant vector fields, meaning the existence and stability of branches of (relative) periodic solutions in generic Hopf bifurcation, 
taking into account the dependence on Taylor coefficients of the vector field. Similarly, we speak of branching patterns of (relative) invariant circles for $\Delta$-equivariant diffeomorphisms.

Theorem 3.4 The branching patterns of (relative) invariant circles for nonresonant (or weakly resonant) Hopf bifurcations from a $\Delta$-symmetric fixed point for $\Delta$-equivariant maps are identical to the branching patterns of (relative) periodic solutions for Hopf bifurcations from a $\Delta$-symmetric equilibrium for $\Delta$-equivariant vector fields.

\subsection{Interpretation of symmetries}

Let $C=C_{\lambda}$ be a bifurcating invariant circle for the diffeomorphism $f$. Alternatively, we can view $C$ as a periodic solution for the vector field $h$. We have already introduced the isotropy subgroup $J \subset \Delta \times S^{1}$ which fixes $C$ pointwise. Since $S^{1}$ lies in the centre of $\Delta \times S^{1}$, it follows that $S^{1}$ lies in the normaliser $N(J)$ of $J$. Hence Fix $J$ is $S^{1}$-invariant.

Proposition 3.5 Suppose that $C$ is an invariant circle with isotropy subgroup $J \subset \Delta \times S^{1}$ with $\operatorname{dim} N(J)=\operatorname{dim} J+1$. Then generically the subgroup of $\Delta \times S^{1}$ that fixes $C$ setwise is the group generated by $J$ and $S^{1}$.

Proof It follows from general theory that $C$ is generically an isolated dynamically-invariant set in Fix $J$. Hence $S^{1}$ must fix $C$ setwise.

On the other hand, $S^{1}$ acts freely and therefore transitively on $C$. So if $g \in \Delta \times S^{1}$ and $g x \in C$ for some $x \in C$, then $g x=\theta x$. Therefore $\theta^{-1} g \in J$ and so $g \in\left\langle J, S^{1}\right\rangle$.

Remark 3.6 The condition $\operatorname{dim} N(J)=\operatorname{dim} J+1$ is not restrictive and is immediate for $\mathbb{C}$-axial isotropy subgroups. Otherwise, $\operatorname{rank} N(J) / J=d \geq 2$, where $\operatorname{rank} G$ of a compact Lie group $G$ is the dimension of the maximal torus in $G$. The invariant circle is then replaced by an invariant torus $\mathbb{T}^{d}$ (actually a relative invariant circle) for the diffeomorphism $f$, and the setwise symmetry group of $\mathbb{T}^{d}$ is of dimension strictly larger than $\operatorname{dim} J+1$.

Define $\Delta_{C}=J \cap \Delta$ and $\Sigma_{C}=\pi(J)$ where $\pi: \Delta \times S^{1} \rightarrow \Delta$ is projection. Note that $\Delta_{C} \subset \Sigma_{C} \subset \Delta$, and that $\Delta_{C}$ is the largest subgroup of $\Delta$ that fixes $C$ pointwise whereas $\Sigma_{C}$ is the largest subgroup of $\Delta$ that fixes $C$ setwise.

We distinguish the cases $\Sigma_{C} / \Delta_{C} \cong S^{1}$ and $\Sigma_{C} / \Delta_{C} \cong \mathbb{Z}_{r}$. In the former case, $C$ is a relative equilibrium for the vector field $h$ and $f: C \rightarrow C$ is a rigid rotation (typically irrational). The continuous $S^{1}$ symmetry prohibits phaselocking, and the rotation number of $f$ varies smoothly on parameters.

In the case $\Sigma_{C} / \Delta_{C} \cong \mathbb{Z}_{r}$, we have a $\left(\Delta_{C}, \Sigma_{C}\right)$-symmetric periodic solution $C$ for the vector field $h$. The dynamics on $C$ for the diffeomorphism $f$ is discussed in detail in Subsection 3.4.

\subsection{Dynamics on the invariant circles}

Another advantage of the approach in Subsection 3.2 is that (even when $\Delta=\mathbf{1}$ ), approximation by an equivariant vector field $h$ is the natural way of studying the dynamics on 
the invariant circles $C_{\lambda}$. The relevant results for the case $\Delta=\mathbf{1}$ can be found for example in $[2,3]$. We assume some familiarity with the case $\Delta=\mathbf{1}$ (referring to $[2,3]$ for details), and concentrate instead on the differences that arise for general $\Delta$.

Let $C=C_{\lambda}$ be an invariant circle for the $\Delta$-equivariant diffeomorphism $f$ and define $\Delta_{C} \subset \Sigma_{C} \subset \Delta$ as in Subsection 3.3. We assume that $\Sigma_{C} / \Delta_{C} \cong \mathbb{Z}_{r}$. Generically, the dynamics is Morse-Smale and consists of periodic sinks, periodic sources, and trajectories connecting these periodic solutions. However, the open and dense set of Morse-Smale dynamics on such invariant circles turns out to be small in the measure-theoretic sense near to the bifurcation, and the full picture is rather delicate.

Two parameters are required to describe the bifurcation: the bifurcation parameter $\lambda$ and the frequency $\omega$ appearing in $(d f)_{0,0}=e^{2 \pi i \omega}$. For each rational $p / q$ (written in its lowest terms), there is an Arnold tongue $A_{p / q}$ in parameter space emanating from the point $(\lambda, \omega)=(0, p / q)$. This is the region of parameter space in which $f$ has rational rotation number $p / q$.

Phaselocking For $(\lambda, \omega) \in A_{p / q}$, the asymptotic dynamics is generically phaselocked and consists of periodic sink/source pairs of period $q$ (see Section 2). Obviously, they have spatial symmetry $\Delta_{\text {per }}=\Delta_{C}$. By Proposition 2.1 , the spatiotemporal symmetry $\Sigma_{\text {per }}$ satisfies

$$
\Sigma_{\text {per }} / \Delta_{\text {per }} \cong \mathbb{Z}_{s} \text { where } s=\operatorname{gcd}(q, r) .
$$

The phaselocked periodic points $x$ have absolute period $q$ and relative period $q / s$. By Proposition 2.2, $f^{q / s}(x)=\sigma_{C}^{p r / s} x$ where $\sigma_{C} \in \Sigma_{C}$ is chosen (uniquely $\bmod \Delta_{C}$ ) so that $\sigma_{C} x=e^{2 \pi i / r} x$ for all $x \in C$.

Proposition 3.7 Let $C_{\lambda}$ be a branch of invariant circles and suppose that $\Sigma_{C} / \Delta_{C} \cong \mathbb{Z}_{r}$. Suppose that $(\lambda, \omega) \in A_{p / q}(\lambda$ small $)$. Let $s=\operatorname{gcd}(q, r)$. Then generically, there are $D r / s$ periodic sinks and $\mathrm{Dr} / \mathrm{s}$ periodic sources, for some positive integer $D \geq 1$.

Proof The linearisation $(d h)_{0}=e^{2 \pi i p / q}$ gives rise to $\mathbb{Z}_{q}$ Birkhoff normal form symmetry (for any $r$ ). We claim that this symmetry, when coupled with $\mathbb{Z}_{r}$-equivariance, leads to $\mathbb{Z}_{Q}$ Birkhoff normal form symmetry where $Q=q r / s$.

To prove the claim, observe that the combined effect of the $\mathbb{Z}_{q}$ Birkhoff normal form symmetry together with $\mathbb{Z}_{r}$-equivariance yields symmetries of the form

$$
z \mapsto e^{2 \pi i\left\{\frac{a}{q}+\frac{b}{r}\right\}} z=e^{2 \pi i(a r+b q) / q r} z
$$

where $a, b \in \mathbb{Z}$. But $a r+b q$ is a general multiple of $s=\operatorname{gcd}(q, r)$. Hence, we have symmetries of the form

$$
z \mapsto e^{2 \pi i c s / q r} z=e^{2 \pi i c / Q} z,
$$

where $c \in \mathbb{Z}$, proving the claim.

It follows that the number of points that lie on periodic sinks (or sources) is given by $D Q$ for some $D \geq 1$. Since each periodic orbit contains precisely $q$ points, we obtain $D Q / q$ periodic sinks (or sources) as required. 
We note that there is a single $\Sigma_{C}$-group orbit of sink/source pairs of periodic solutions on the invariant circle if and only if $D=1$.

Remark 3.8 In the nonequivariant context, $\Delta=1$, stronger results are known. Consider an Arnold tongue $A_{p / q}$, where $q \geq 5$. (We do not consider the strongly resonant cases $q \leq 4$ here.) Generically, it is the case that for each $(\lambda, \omega) \in A_{p / q}$ (with $\lambda$ close enough to 0 ), there is a single sink-source pair of periodic solutions on the invariant circle (see [3, Theorem 5.4.1]). In other words, $D=1$ in Proposition 3.7.

There are a number of problems in formulating the corresponding result for equivariant systems. First of all, the proof in the nonequivariant case relies strongly on the fact that the centre manifold is two-dimensional. In the presence of symmetry, the centre manifold may be higher-dimensional, so it is necessary to immediately restrict attention to invariant circles corresponding to $\mathbb{C}$-axial isotropy subgroups $J \subset \Delta \times S^{1}$. But since we are in a $p / q$-resonant tongue, the symmetry is immediately broken to $J_{q} \subset \Delta \times \mathbb{Z}_{q}$ where $J_{q}=J \cap\left(\Delta \times \mathbb{Z}_{q}\right)$. Hence, we require the stronger assumption that $J_{q}$ is a $\mathbb{C}$-axial subgroup of $\Delta \times \mathbb{Z}_{q}$ (so $\operatorname{dim} \operatorname{Fix} J_{q}=2$ ).

Even under the assumption that $J_{q}$ is $\mathbb{C}$-axial, we cannot always conclude that $D=1$ due to the possibility of hidden symmetries [10]. As in the proof of Proposition 3.7, we can write $f$ (in Birkhoff normal form) as $f(z)=e^{2 \pi i p / q} \exp h(z)$, where $h$ is a $\mathbb{Z}_{Q^{-}}$equivariant vector field [2, 3]. This has the form $h(z)=\alpha\left(|z|^{2}, z^{Q}, \bar{z}^{Q}\right) z+\beta\left(|z|^{2}, z^{Q}, \bar{z}^{Q}\right) \bar{z}^{Q-1}, Q=r / s$, where $\alpha$ and $\beta$ are $\mathbb{C}$-valued. To avoid strong resonances, assume that $Q \geq 5$. Suppose that

(i) The real and imaginary parts of the coefficients of $|z|^{2}$ in $\alpha$ are nonzero.

(ii) $\beta(0) \neq 0$.

Then it can be shown (just as in the case $r=1, q \geq 5$, see [3, pages 282-286]) that in the Arnold tongue $A_{p / q}$ the vector field has precisely $Q$ sink-source pairs of equilibria and so $D=1$. Condition (i) is satisfied generically, since $|z|^{2} z$ is the restriction of the 'trivial' $\Delta$-equivariant cubic nonlinearity. (Recall that $\Delta \subset \mathbf{O}(n)$.) However, condition (ii) may fail: it is possible that there are no $\Delta$-equivariant nonlinearities of order $Q-1$ that restrict to the map $\bar{z}^{Q-1}$ on Fix $J_{q}$. For this reason it is possible that generically $D \geq 2$ even when $Q \geq 5$ and $J_{q}$ is $\mathbb{C}$-axial. The integer $D$ depends only on the representation of $\Delta$ on $X$.

If the action of $\Delta$ is two-dimensional, then the difficulties described in Remark 3.8 do not arise. The action of $\Delta$ effectively reduces to a $\mathbb{Z}_{r}$ action and we have the following result:

Proposition 3.9 Suppose that $\Delta=\mathbb{Z}_{r}$ acts faithfully on $\mathbb{R}^{2}$ and that the fixed point at 0 undergoes Hopf bifurcation to an invariant circle $C$. Let $q \geq 1$ and $s=\operatorname{gcd}(q, r)$. If $q r / s \geq 5$, then in the Arnold tongue $A_{p / q}$, generically there are precisely $r / s$ periodic sink/source pairs on $C$.

Remark 3.10 In the nonequivariant setting, the boundaries of the Arnold tongues $A_{p / q}$ with $q \geq 5$ are generically cuspoidal of order $(q-2) / 2$. This is shown by studying the 
$\mathbb{Z}_{q}$-equivariant vector field $h$, see for example [2, p. 311] or [3, Theorem 5.4.1]. These computations carry over to the equivariant context, subject to caveats similar to those in Remark 3.8. Provided the subgroup $J_{q}=J \cap\left(\Delta \times \mathbb{Z}_{q}\right)$ is $\mathbb{C}$-axial, the computation reduces to the two-dimensional fixed point space Fix $J$ and the vector field $\left.h\right|_{\text {Fix } J}$ is $\mathbb{Z}_{Q^{-}}$-equivariant where $Q=q r / s$. Hence we expect that the boundaries of the tongues are cuspoidal of order $(Q-2) / 2$. However, just as in Remark 3.8 , it is necessary to pay attention to hidden symmetries. Certain degeneracies due to hidden symmetries may be tractable using singularity-theoretical ideas such as in [5].

Irrational rotations The union of Arnold tongues $\cup A_{p / q}$ forms an open and dense subset of parameter space. However, Herman [14] has shown that the complement of the union of Arnold tongues $\cup A_{p / q}$ has positive measure, converging to full measure as $\lambda \rightarrow 0$. Moreover, almost everywhere within this complement, the rotation numbers are Diophantine and the dynamics is smoothly conjugate to a rigid irrational rotation. The smooth conjugacy on $C_{\lambda}$ is $\Sigma_{C}$-equivariant by Proposition 2.3 and (being isotopic to the identity) extends to a $\Delta$-equivariant diffeomorphism on $\mathbb{R}^{n}$.

\subsection{Hopf bifurcation from periodic solutions}

We return to Hopf bifurcation from the $(\Delta, \Delta)$-symmetric periodic solution $P$. By the results of this section

Theorem 3.11 The following branching patterns are identical:

- (relative) periodic solutions for Hopf bifurcations from a $\Delta$-symmetric equilibrium for $\Delta$-equivariant vector fields,

- (relative) invariant circles for nonresonant (or weakly resonant) Hopf bifurcations from a $\Delta$-symmetric fixed point for $\Delta$-equivariant maps,

- (relative) two-tori for nonresonant (or weakly resonant) Hopf bifurcations from a $(\Delta, \Delta)$-symmetric periodic solution for $\Delta$-equivariant vector fields.

Let $C=C_{\lambda}$ be a bifurcating periodic solution for the vector field $h$ (equivalently, an invariant circle for the Poincaré map $f$ ) corresponding to an invariant two-torus $\mathbb{T}$ for the underlying flow. Then $\Delta_{C}$ is the largest subgroup of $\Delta$ that fixes $\mathbb{T}$ pointwise, whereas $\Sigma_{C}$ is the largest subgroup of $\Delta$ that fixes $\mathbb{T}$ setwise. If $\Sigma_{C} / \Delta_{C} \cong S^{1}$, then $\mathbb{T}$ is a relative periodic solution (modulated rotating wave) for the underlying flow, and the dynamics on $\mathbb{T}$ is generically a two frequency quasiperiodic flow. In particular, there is no phaselocking.

When $\Sigma_{C} / \Delta_{C} \cong \mathbb{Z}_{r}$ we apply the results of Subsection 3.4. Let $T_{0}$ denote the period of the underlying periodic solution $P$ at $\lambda=0$. The generic flow on $\mathbb{T}$ is phaselocked so that the asymptotic dynamics consists of periodic sink/source pairs, but irrational torus flows occur with arbitrarily high probability near $\lambda=0$. The latter are $\Sigma_{C}$-equivariantly topologically 
equivalent (topologically conjugate up to a time change) to a rigid irrational flow, and the equivalences are almost always smooth.

The Arnold tongue $A_{p / q}$ for $f$ corresponds to torus flows with rational rotation number $p / q$. Let $s=\operatorname{gcd}(q, r)$. Provided $q r / s \geq 5$, the asymptotic dynamics consists of $\mathrm{Dr} / s$ periodic sink/source pairs where $D \geq 1$. In the simplest cases, $D=1$. The absolute and relative periods are

$$
T^{\mathrm{abs}}(\lambda) \sim q T_{0}, \quad T^{\mathrm{rel}}(\lambda)=\frac{1}{s} T^{\mathrm{abs}}(\lambda) \sim \frac{q}{s} T_{0} .
$$

The periodic solutions $y(t, \lambda)$ are $\left(\Delta_{\text {per }}, \Sigma_{\text {per }}\right)$-symmetric where $\Delta_{\text {per }}=\Delta_{C}$ and $\Sigma_{\text {per }} / \Delta_{\text {per }} \cong$ $\mathbb{Z}_{s}$. Moreover, a spatiotemporal generator is given by $\sigma_{C}^{p r / s}$ where $\sigma_{C} \in \Sigma_{C}$ is chosen (uniquely $\left.\bmod \Delta_{C}\right)$ so that $\sigma_{C} x=e^{2 \pi i / r} x$ for all $x \in C$. Hence,

$$
y\left(t+\frac{1}{s} T^{\mathrm{abs}}(\lambda), \lambda\right)=\sigma_{C}^{p r / s} y(t, \lambda)
$$

\section{Hopf bifurcation from discrete rotating waves}

In this section, we consider Hopf bifurcation from a $(\Delta, \Sigma)$-symmetric periodic solution for a $\Sigma$-equivariant flow, where $\Sigma / \Delta$ is finite.

It follows from $[17,18]$ that the Hopf bifurcation reduces to the situation described in Section 3. We sketch the approach in [18] omitting the proofs. (Unfortunately, the notation is somewhat different from that in [18] but this inconvenience seems unavoidable.)

Let $\sigma \in \Sigma$ be a spatiotemporal generator. We can choose $\sigma$ such that $\sigma^{k}$ commutes with the elements of $\Delta$ for some finite $k \geq 1$. Form the abstract group $\Delta_{0}=\Delta \rtimes \mathbb{Z}_{k}$ by adjoining to $\Delta$ an element $\tau$ of order $k$ such that $\tau \delta \tau^{-1}=\sigma \delta \sigma^{-1}$ for $\delta \in \Delta$.

Suppose that the periodic solution undergoes a nonresonant Hopf bifurcation (so the eigenvalues of the Poincaré map are irrational). Through arbitrarily high order, the entire bifurcation analysis reduces as in Section 3 to Hopf bifurcation from an equilibrium for a $\Delta_{0^{-}}$ equivariant vector field $h$. This is a special case of [18, Theorem 2.1] (using the simplification in $[18$, Remark 2.2(b)]). Once again, there is a notion of weak resonance which enables such a reduction through "sufficiently high order".

For aspects of the bifurcation that are determined at finite order, the results of Sections 3.1-3.3 apply directly (subject to interpretations). For example, we can apply Theorem 3.1 by considering $\mathbb{C}$-axial isotropy subgroups $J$ of the group $\Delta_{0} \times S^{1}$. This was done in $[18$, Section 3.2]. More generally, we obtain the following analogue to Theorem 3.11:

Theorem 4.1 Define $\Delta_{0}=\Delta \rtimes \mathbb{Z}_{k}$ as above. The following branching patterns are identical:

- (relative) periodic solutions for Hopf bifurcations from a $\Delta_{0}$-symmetric equilibrium for $\Delta_{0}$-equivariant vector fields,

- (relative) two-tori for nonresonant (or weakly resonant) Hopf bifurcations from a $(\Delta, \Sigma)$-symmetric periodic solution for $\Sigma$-equivariant vector fields. 
As in Section 3, we can relate the symmetry properties of relative periodic solutions for the $\Delta_{0}$-equivariant vector fields to the symmetry properties of the corresponding invariant two-tori for the $\Sigma$-equivariant vector fields. Here, we follow [18, Section 2.4] but with slight modifications in the notation to conform with Section 3.

Let $C=C_{\lambda}$ be a bifurcating periodic solution for the vector field $h$ corresponding to an invariant two-torus $\mathbb{T}$ for the underlying flow. We define the spatial and spatiotemporal symmetry groups $\Delta_{C} \subset \Sigma_{C} \subset \Delta_{0}$ just as in Section 3. Let $\Delta_{\mathbb{T}}=\Delta_{C} \cap \Delta$ and define $\Sigma_{\mathbb{T}}$ to be the group generated by $\Sigma_{C} \cap \Delta$ and $\sigma^{d} \delta$. Here, $d \geq 1$ least and $\delta \in \Delta$ are chosen such that $\tau^{d} \delta \in \Sigma_{C}$. Then $\Delta_{\mathbb{T}}$ is the largest subgroup of $\Delta$ that fixes $\mathbb{T}$ pointwise and $\Sigma_{\mathbb{T}}$ is the largest subgroup of $\Delta$ that fixes $\mathbb{T}$ setwise. If $\Sigma_{C} / \Delta_{C} \cong S^{1}$, then $\mathbb{T}$ is a relative periodic solution. As usual, we focus on the case $\Sigma_{C} / \Delta_{C} \cong \mathbb{Z}_{r}, r \geq 1$.

Irrational rotations To discuss the dynamics on the invariant tori, it is necessary to introduce the first hit pull-back map $f=\sigma^{-1} g^{(1)}$ defined on a $\Delta$-invariant cross-section to $P$. We refer to [18] for details, but note the relation $f=\tau^{-1} \exp h$ through arbitrarily high order. Also, note that $f$ is $\Delta$-twisted equivariant in the sense that $f \delta=\phi(\delta) f$ where $\phi$ is the automorphism $\phi(\delta)=\sigma^{-1} \delta \sigma$ for $\delta \in \Delta$.

Since $C$ is an invariant circle for $h$, and $f=\tau^{-1} \exp h$ where $\tau$ has order $k$ and commutes with $h$, it follows that $C$ is an invariant circle for $f^{k}$. However $f$ need not preserve $C$. Let $N \geq 1$ be least such that $f^{N}(C) \subset C$. Then $f^{N}$ is $\mathbb{Z}_{r^{-}}$-twisted equivariant, and hence $\mathbb{Z}_{r^{-}}$ equivariant by Proposition 2.5. When $f^{N}: C \rightarrow C$ has irrational rotation number $\omega$ (i.e. the underlying flow on the corresponding two-torus has irrational rotation number), it follows from Corollary 2.4 that $f^{N}$ is $\mathbb{Z}_{r}$-equivariantly topologically conjugate to a rigid rotation. Again, it is almost always the case that the conjugacy is smooth. For each $C_{j}=f^{j}(C)$, $j=0, \ldots, N-1$, we can choose a (typically smooth) conjugacy $\psi_{j}: C_{j} \rightarrow C_{j}$ such that $\left.\psi_{j}\left(f^{N}\right)\right|_{C_{j}} \psi_{j}^{-1}=R_{\omega}$. Altogether, we obtain a conjugacy $\psi$ defined on $C_{0} \cup \cdots \cup C_{N-1}$ with the property that $\psi f^{N} \psi^{-1}=R_{\omega}$. We can modify the choices of $\psi_{j}$ so that $\psi$ is $\Sigma_{\mathbb{T}^{-}}$-equivariant. Finally, $\psi$ is typically smooth and extends to a $\Sigma$-equivariant diffeomorphism on $\mathbb{R}^{n}$.

Phaselocking Again, the generic flow on $\mathbb{T}$ has rational rotation number and phaselocked periodic solutions as in Section 3. Following [18], we associate the rotation number $p / q$ to $L_{0}^{k}$ where $L_{0}$ is the linearisation of the map first-hit pull-back map $f$ mentioned above. So $L_{0}^{k}=e^{2 \pi i p / q}$. The number $\ell$ in [18] corresponds to the denominator $q$. Thus we form the abstract group $\Delta \rtimes \mathbb{Z}_{k q}$ by adjoining an element $\tau$ of order $k q$ to $\Delta$, where $\tau$ induces the same automorphism as before: $\tau^{-1} \delta \tau=\phi(\delta)=\sigma^{-1} \delta \sigma$.

The representation of the abstract group $\Delta \rtimes \mathbb{Z}_{k q}$ is generated by the actions of $\Delta$ and $L_{0}$ where $\tau$ is identified with $L_{0}^{-1}$. In Birkhoff normal form, $f$ can be written as $f=L_{0} \exp h$ where $h$ is a $\Delta \rtimes \mathbb{Z}_{k q}$-equivariant vector field satisfying $h(0)=0$ and $(d h)_{0}=0$.

In the weakly resonant case, the dynamics in the Arnold tongue $A_{p / q}$ consists generically of phaselocked solutions. Note that phaselocked periodic solutions on $\mathbb{T}$ (or $C$ ) correspond to equilibria for the $\Delta \rtimes \mathbb{Z}_{k q}$-equivariant vector field $h: C \rightarrow C$ and such equilibria have isotropy $J_{q} \subset \Delta \rtimes \mathbb{Z}_{k q}$. By [18, Proposition 2.8], the phaselocked solutions have spatial 
symmetry $\Delta_{\text {per }}=J_{q} \cap \Delta$ and spatiotemporal symmetry $\Sigma_{\text {per }}$ generated by $\Delta_{\text {per }}$ and $\sigma^{b} \delta$ where $\tau^{b} \delta \in J_{q}$ for some $b \geq 1$ and $\delta \in \Delta$ with $b$ least. Write $\Sigma_{\text {per }} / \Delta_{\text {per }} \cong \mathbb{Z}_{s}$ where $s \geq 1$.

By [18, Proposition 2.10], the phaselocked solutions $y(t)$ have absolute and relative periods

$$
T^{\mathrm{abs}}(\lambda) \sim b s T_{0}^{\mathrm{rel}}, \quad T^{\mathrm{rel}}(\lambda)=\frac{1}{s} T^{\mathrm{abs}}(\lambda) \sim b T_{0}^{\mathrm{rel}},
$$

where $T_{0}^{\text {rel }}$ is the relative period of the underlying periodic solution. Moreover

$$
y\left(t+\frac{1}{s} T^{\mathrm{abs}}(\lambda), \lambda\right)=\sigma^{b} \delta y(t, \lambda) .
$$

Remark 4.2 When $k=1$, it can be verified directly that $b=q / s, s=\operatorname{gcd}(q, r)$ and $\sigma^{b} \delta=\sigma_{C}^{p r / s}$. Hence these calculations are consistent with the results in Section 3 .

Remark 4.3 As in Remarks 3.8 and 3.10, it is often possible to obtain results on the number of phaselocked periodic solutions and the structure of the Arnold tongues by studying the vector field $h$. Again, it is necessary to assume $\mathbb{C}$-axiality and to pay attention to hidden symmetries.

\section{Hopf bifurcation from relative periodic solutions}

In this section, we briefly consider Hopf bifurcation from a $(\Delta, \Sigma)$-symmetric relative periodic solution $P$ for a $\Gamma$-equivariant flow. It is assumed that $\Delta$ is compact and that $\Gamma$ is an algebraic group acting properly on a compact manifold. (The condition that $\Gamma$ is algebraic is satisfied by compact Lie groups and Euclidean groups.) It follows from $[24,18]$ that certain aspects of the Hopf bifurcation reduce to the situation described in Section 3. We sketch this approach omitting the proofs. (Again, the notation is unavoidably different from that in $[24,18]$.)

Let $\sigma \in \Sigma$ be a spatiotemporal generator. We can again choose $\sigma$ so that $\sigma^{k}$ commutes with elements of $\Delta$ for some finite $k \geq 1$. Form the abstract group $\Delta_{0}=\Delta \rtimes \mathbb{Z}_{k}$ by adjoining to $\Delta$ an element $\tau$ of order $k$ such that $\tau \delta \tau^{-1}=\sigma \delta \sigma^{-1}$ for all $\delta \in \Delta$.

Suppose that the relative periodic solution undergoes a nonresonant (or weakly resonant) Hopf bifurcation. Through arbitrarily high (or sufficiently high) order and modulo drifts along the $\Gamma$ group orbit, the entire bifurcation analysis reduces to Hopf bifurcation from an equilibrium for a $\Delta_{0}$-equivariant vector field $h$. This is a special case of $[24,18]$. Combining [18, Theorem 8.1] and the methods of Sections 3.1-3.3 we obtain (subject to interpretations)

Theorem 5.1 Let $\Gamma$ be an algebraic group and $\Delta$ a compact subgroup. Define $\Delta_{0}=\Delta \rtimes \mathbb{Z}_{k}$ as above. Modulo drifts along the $\Gamma$ group orbit the following branching patterns are identical:

- (relative) periodic solutions for Hopf bifurcations from a $\Delta_{0}$-symmetric equilibrium for $\Delta_{0}$-equivariant vector fields,

- relative two-tori for nonresonant (or weakly resonant) Hopf bifurcations from a $(\Delta, \Sigma)$ symmetric relative periodic solution for $\Gamma$-equivariant vector fields. 
In contrast to previous sections of this paper, Theorem 5.1 is not sufficient for issues such as phaselocking and irrational rotations. It is necessary to first reduce as in [24] to a skew product system as follows.

By [24, Proposition 1.2], the spatiotemporal generator $\sigma \in \Sigma$ can be chosen so that $\sigma=\alpha \exp \eta=(\exp \eta) \alpha$, where $\alpha \in \Gamma$ has finite order $n \geq k$ and $\eta \in L Z(\Sigma)$ lies in the Lie algebra of the centraliser of $\Sigma$. Define $\Sigma^{\prime}=\Delta \rtimes \mathbb{Z}_{n}$ by adjoining to $\Delta$ an element $Q$ of order $n$. Let $V$ be a $\Delta$-invariant cross-section to $\Gamma P$ and let $S^{1}=\mathbb{R} / n \mathbb{Z}$. Define $X=V \times S^{1}$ and denote the Lie algebra of $\Gamma$ by $L \Gamma$. Then we obtain a skew product on $\Gamma \times X$

$$
\dot{\gamma}=\gamma F_{\Gamma}(x), \quad \dot{x}=F_{X}(x)
$$

where $F_{\Gamma}: X \rightarrow L \Gamma, F_{X}: X \rightarrow T X$ are smooth vector fields. The construction can be done so that $V$ is $\Sigma^{\prime}$-invariant and $F_{\Gamma}, F_{X}$ are $\Sigma^{\prime}$-equivariant with respect to the action

$$
\delta \cdot(\gamma, v, \theta)=\left(\gamma \delta^{-1}, \delta v, \theta\right), \quad \delta \in \Delta, \quad Q \cdot(\gamma, v, \theta)=\left(\gamma \alpha^{-1}, Q v, \theta+1\right) .
$$

The underlying relative periodic solution reduces to a $\left(\Delta, \Sigma^{\prime}\right)$-symmetric periodic solution for $F_{X}$. In particular, the $\Sigma^{\prime}$-equivariant vector field $F_{X}: X \rightarrow T X$ can now be studied exactly as in Section 4. A complete characterisation of the symmetry properties of phaselocked solutions can be obtained in this way. However, in general the irrational rotation case is more difficult.

Remark 5.2 In Section 6, we study the irrational rotation case in a simple situation with $\Gamma=\mathbf{S E}(2)$. (Many of the technicalities in [24] are not present for this example.) The reader can easily see from the analysis presented there that the case of trivial spatial symmetry $\Delta=1$ "reduces" to the question of transforming an $L \Gamma$-valued map (cocycle) over an irrational torus flow to a constant, which is a difficult open problem (see [15] for the case $\Gamma=\mathbf{S U}(2))$.

\section{Application to $\ell$-armed spirals}

In this section, we consider Hopf bifurcation from a relative periodic solution with $\Gamma=\mathbf{S E}(2)$, $\Sigma=\mathbf{S O}(2)$ and $\Delta=\mathbb{Z}_{\ell}, \ell \geq 1$. This situation arises in the context of spiral waves in planar excitable media. In the first instance, spiral waves are rigidly rotating relative equilibria (rotating waves) and an initial Hopf bifurcation leads to relative periodic solutions (modulated rotating waves). We are interested in secondary Hopf bifurcations from such relative periodic solutions.

The case of single-armed spirals $(\ell=1)$ was partially analysed previously by Sandstede et al. [23]. We complete their analysis and extend the results to the case of multi-armed spirals $(\ell>1)$.

Remark 6.1 The first step, as in Sandstede et al. [23], is to reduce to an equivariant flow on a compact manifold (namely the setup in Section 5). For technical reasons related to 
continuous spectrum issues, the required spectral hypothesis [23, Hypothesis (S)] fails for nonlocalised spiral waves. Nevertheless, following [23] we proceed as if the reduction is valid, referring to [23, Section 6] and [22] for a discussion and resolution of these issues.

Let $T_{0}$ denote the relative period of the underlying relative periodic solution. We write the spatiotemporal generator $\sigma \in \mathbf{S O}(2)$ as $\sigma=\exp \eta$ where $\eta=2 \pi i \omega_{\text {rot }} T_{0} \in L \mathbf{S O}(2)$, (so $\omega_{\text {rot }} \in \mathbb{R}$ ). In particular, $k=n=1, \alpha=\mathrm{id}$ and $Q=\mathrm{id}$ in Section 5. By Theorem 5.1,

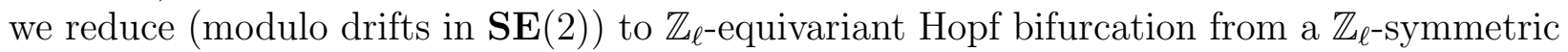
equilibrium. By [12], generically there is a two-dimensional centre manifold $V \cong \mathbb{C}$ and either $\mathbb{Z}_{\ell}$ acts trivially on $V$ (the non-symmetry-breaking case) or $V$ is a nontrivial irreducible representation of $\mathbb{Z}_{\ell}$. We focus on the latter, so $\mathbb{Z}_{\ell}$ acts on $V \cong \mathbb{C}$ by

$$
R_{2 \pi / \ell} \cdot v=e^{2 \pi i m / \ell} v
$$

for some $m=1, \ldots,[\ell / 2]$. The kernel of the action is $\mathbb{Z}_{d}$ where $d=\operatorname{gcd}(\ell, m)$. Effectively, we obtain an action of $\mathbb{Z}_{r}$ on $V$ where $r=\ell / d$.

Since $V$ is two dimensional, there is a unique $\mathbb{C}$-axial isotropy subgroup $J \subset \mathbb{Z}_{\ell} \times S^{1}$ given by $J=\mathbb{Z}_{\ell}$ generated by $\left(R_{2 \pi / \ell},-2 \pi m / \ell\right)$. As in previous sections, generically there is a unique branch of periodic solutions/invariant circles $C$ for the $\mathbb{Z}_{\ell^{-}}$equivariant vector field/diffeomorphism. Here, $C$ is fixed pointwise by $\Delta_{C}=J \cap \mathbb{Z}_{\ell}=\mathbb{Z}_{d}$ and setwise by $\Sigma_{C}=\mathbb{Z}_{\ell}$. In the $\mathbf{S E}(2)$-equivariant equations, we obtain a unique branch of relative invariant two-tori $\mathbb{T}$ that are fixed pointwise by $\mathbb{Z}_{d}$. (We caution that $\mathbb{T}$ is not itself necessarily a torus, but $\mathbb{T} / \mathbf{S E}(2)$ is a two-torus.)

In Sections 3.4 and 3.5, we identified an element $\sigma_{C} \in \Sigma_{C}$ that acts as $z \mapsto e^{2 \pi i / r} z$ on $C$. It is easily verified that the abstract symmetry $\sigma_{C}$ corresponds to the physical symmetry $\left(R_{2 \pi / \ell}\right)^{a}$ where $a$ is the least positive integer such that $a m \equiv d \bmod \ell$ or equivalently $a m / d \equiv$ $1 \bmod r$.

Dynamics on the invariant relative two-torus $\mathbb{T}$ By $[24$, Theorem 1.3(b)], the $\mathbf{S E}(2)$ equivariant dynamics are governed by skew-product equations (5.1) with $\Sigma^{\prime}=\Delta=\mathbb{Z}_{\ell}$. Write $\mathbf{S E}(2)=\mathbf{S O}(2) \ltimes \mathbb{C}, \gamma=(\varphi, p)$, where $\varphi \in \mathbf{S O}(2)$ is rotation and $p \in \mathbb{C}$ is translation. We obtain the skew-product system on $\mathbf{S E}(2) \times X$, where $X=V \times S^{1}$, given by

$$
\dot{p}=e^{i \varphi} F_{p}(x), \quad \dot{\varphi}=F_{\varphi}(x), \quad \dot{x}=F_{X}(x),
$$

subject to the $\mathbb{Z}_{\ell^{-} \text {equivariance conditions }}$

$$
F_{p}\left(R_{2 \pi / \ell} \cdot x\right)=R_{2 \pi / \ell} F_{p}(x), \quad F_{\varphi}\left(R_{2 \pi / \ell} \cdot x\right)=F_{\varphi}(x), \quad F_{X}\left(R_{2 \pi / \ell} \cdot x\right)=R_{2 \pi / \ell} \cdot F_{X}(x) .
$$

The underlying rotating wave reduces to a $\left(\mathbb{Z}_{\ell}, \mathbb{Z}_{\ell}\right)$-symmetric periodic solution for $F_{X}$ with relative and absolute period $T_{0}$.

After centre manifold reduction, the $\mathbb{Z}_{\ell}$ representation $V \cong \mathbb{C}$ can be taken to be a cross-section to the periodic solution for $F_{X}$. Introducing the bifurcation parameter $\lambda$, let $f: V \times \mathbb{R} \rightarrow V$ be the Poincaré map so $f$ is $\mathbb{Z}_{\ell^{-}}$-equivariant with fixed point 0 . The eigenvalues of $(d f)_{0,0}$ are complex conjugate eigenvalues on the unit circle, denoted $e^{ \pm 2 \pi i \omega T_{0}}$. 
By Sections 3.4 and 3.5, we have the following result on phaselocking for $F_{X}$. Recall that $A_{p / q}$ is the Arnold tongue emanating from $(0, p / q)$ in $(\lambda, \omega)$ parameter space.

Proposition 6.2 Suppose that $(\lambda, \omega) \in A_{p / q}(\lambda$ small). Let $s=\operatorname{gcd}(q, r)$ and suppose (to avoid strong resonance) that $q r / s \geq 5$. Then generically there are precisely $r / s$ sink-source pairs of periodic solutions for $F_{X}$ (of absolute period $T^{\mathrm{abs}}(\lambda) \sim q T_{0}$ and relative period $T^{\mathrm{rel}}(\lambda) \sim \frac{q}{s} T_{0}$ ) on the invariant torus. Moreover, each periodic solution $y(t, \lambda)$ has spatial symmetry $\Delta_{\text {per }}=\mathbb{Z}_{d}$ and spatiotemporal symmetry $\Sigma_{\text {per }}=\mathbb{Z}_{s d}$, and satisfies

$$
y\left(t+\frac{1}{s} T^{\mathrm{abs}}(\lambda), \lambda\right)=\left[R_{2 \pi / \ell}\right]^{\text {apr } / s} \cdot y(t, \lambda) .
$$

Noncompact drift on the phaselocked relative periodic solutions Next, we consider the dynamics on the relative periodic solutions $u(t, \lambda)$ for the underlying $\mathbf{S E}(2)$-equivariant flow corresponding to the phaselocked periodic solutions $y(t, \lambda)$ in Proposition 6.2. We expect either bounded or linear drift along $\mathbf{S E}(2)$ group orbits [4] depending on the spatiotemporal symmetry of the bifurcating relative periodic solutions.

When $d \geq 2$, we have $Z\left(\Delta_{\text {per }}\right)=\mathbf{S O}(2)$ and the drift is compact.

We now concentrate on the case $d=1$ so that $Z\left(\Delta_{\text {per }}\right)=\mathbf{S E}(2)$, and there is the possibility of noncompact drift. We have $r=\ell, s=\operatorname{gcd}(\ell, q)$, and $a m \equiv 1 \bmod \ell$.

Proposition 6.3 Suppose that $d=1$ and let $(\lambda, \omega) \in A_{p / q}$. A necessary condition for noncompact drift on the corresponding phaselocked relative periodic solutions near $\lambda=0$ is that

$$
q \omega_{\text {rot }} T_{0}+a p \equiv 0 \bmod s .
$$

Proof By [24],

$$
u\left(\frac{1}{s} T^{\mathrm{abs}}(\lambda), \lambda\right)=\gamma(\lambda)\left[R_{2 \pi / \ell}\right]^{a p \ell / s} \cdot u(0, \lambda),
$$

where $\gamma(\lambda)$ is a general element of $Z\left(\Delta_{\text {per }}\right)$ close to the rotation $R_{\frac{2 \pi q}{s} \omega_{\text {rot }} T_{0}}$. In particular, $\gamma(\lambda)\left[R_{2 \pi / \ell}\right]^{a p \ell / s} \sim R_{\frac{2 \pi q}{s} \omega_{\text {rot }} T_{0}+2 \pi a p / s}$. Noncompact drift occurs only when this group element is a translation, necessarily the identity. The result follows.

We end the discussion of phaselocking by stating the precise condition for noncompact drift at a given value of the bifurcation parameter $\lambda$. Express the $\dot{\varphi}$ equation (which is $\frac{1}{s} T^{\mathrm{abs}}(\lambda)$-periodic) as a Fourier series and define $2 \pi \omega_{\text {rot }}(\lambda)$ to be the constant term. Then noncompact drift occurs generically if and only if

$$
\omega_{\mathrm{rot}}(\lambda) T^{\mathrm{abs}}(\lambda)+a p \equiv 0 \bmod s .
$$

Noncompact drift on irrational torus flows Finally, we consider the possibility of noncompact drift in regions of parameter space corresponding to irrational rotation numbers. As described earlier, this region has measure converging to full measure as $\lambda \rightarrow 0$. From now on, we denote the invariant two-torus for $F_{X}$ by $\mathbb{T}^{2}$ and we use angular coordinates $\left(\theta_{1}, \theta_{2}\right) \in \mathbb{T}^{2}$. 
Proposition 6.4 Within the region of irrational rotation number, near $\lambda=0$, there is a $\mathbb{Z}_{\ell}$-equivariant change of coordinates after which the skew product (6.1) becomes

$$
\dot{p}=e^{i \varphi} F_{p}\left(\theta_{1}, \theta_{2}\right), \quad \dot{\varphi}=\omega_{0}+F_{\varphi}\left(\theta_{1}\right), \quad \dot{\theta}_{1}=\omega_{1}, \quad \dot{\theta}_{2}=\omega_{2},
$$

where $F_{\varphi}: \mathbb{T}^{1} \rightarrow \mathbb{R}, F_{p}: \mathbb{T}^{2} \rightarrow \mathbb{C}$ are continuous functions with $\int_{\mathbb{T}^{1}} F_{\varphi} d \theta_{1}=0$.

Proof At $\lambda=0$, we have $\dot{\varphi} \equiv \omega_{\text {rot }} \neq 0$, so for $\lambda$ close to zero we can smoothly reparameterise time so that $\dot{\varphi} \equiv \omega_{0}$. The flow on the invariant torus $\mathbb{T}^{2}$ for $F_{X}$ possesses a $\mathbb{Z}_{\ell}$-invariant cross-section $\mathbb{T}^{1}$ (coordinatised by $\theta_{1}$ ) such that the Poincaré map $f: \mathbb{T}^{1} \rightarrow \mathbb{T}^{1}$ has irrational rotation number. By a $\mathbb{Z}_{\ell^{-}}$equivariant change of coordinates (Corollary 2.4) we can arrange that $f$ is a rigid rotation. The return time $\tau=\tau\left(\theta_{1}\right)$ is $\mathbb{Z}_{\ell}$-invariant. Reparametrising time once again so that $\tau$ is constant, we obtain equations (6.3).

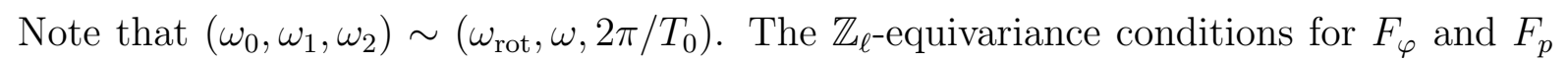
take the form

$$
F_{\varphi}\left(\theta_{1}+2 \pi m / \ell\right)=F_{\varphi}\left(\theta_{1}\right), \quad F_{p}\left(\theta_{1}+2 \pi m / \ell, \theta_{2}\right)=e^{2 \pi i / \ell} F_{p}\left(\theta_{1}, \theta_{2}\right) .
$$

We emphasise three possibilities for the drift on the relative invariant torus:

- Linear drift: $p(t)=c t+o(t)$ as $t \rightarrow \infty$, where $c \neq 0$.

- Sublinear drift: $p(t)=o(t)$ as $t \rightarrow \infty$. That is, $p(t) / t \rightarrow 0$ as $t \rightarrow \infty$.

- Bounded drift: $p(t)$ is bounded.

In particular, we give necessary and sufficient conditions for linear and sublinear drift (generalising [23]) and sufficient conditions for bounded drift (following [20]).

As in the case of phaselocked flows, the drift is automatically bounded if $d \geq 2$ since the equivariance restrictions imply that $F_{p} \equiv 0$. Hence, we suppose from now on that $d=1$.

Proposition 6.5 Suppose that $\ell \geq 1$ and that $d=1$. Let $a \geq 1$ be least such that am $\equiv$ $1 \bmod \ell$. If there are integers $k_{1}, k_{2}$ such that

$$
\omega_{0}+\left(a+k_{1} \ell\right) \omega_{1}+k_{2} \omega_{2}=0
$$

is satisfied then generically (provided a certain Fourier coefficient is nonzero) there is linear drift on the relative torus. Otherwise, the drift is sublinear.

Proof Without loss, we consider the initial condition $\left(p, \varphi, \theta_{1}, \theta_{2}\right)_{0}=(0,0,0,0)$. Define $F\left(\varphi, \theta_{1}, \theta_{2}\right)=e^{i \varphi} F_{p}\left(\theta_{1}, \theta_{2}\right)$. We are interested in the growth of the expression

$$
\left.p(t)=\int_{0}^{t} F\left(\varphi(s), \omega_{1} s, \omega_{2} s\right)\right) d s .
$$

Note that $\varphi(t)=\omega_{0} t+G\left(\omega_{1} t\right)$ where $G: \mathbb{T}^{1} \rightarrow \mathbb{R}$ is given by $G\left(\theta_{1}\right)=\omega_{1}^{-1} \int_{0}^{\theta_{1}} F_{\varphi}\left(\theta_{1}^{\prime}\right) d \theta_{1}^{\prime}$. 
First suppose that $\omega_{0}$ is rationally independent of $\omega_{1}$ and $\omega_{2}$. We introduce a new variable $\theta_{0}$ and consider the uniquely ergodic flow on $\mathbb{T}^{3}$ given by

$$
\dot{\theta}_{0}=\omega_{0}, \quad \dot{\theta}_{1}=\omega_{1}, \quad \dot{\theta}_{2}=\omega_{2} .
$$

Define $H\left(\theta_{0}, \theta_{1}, \theta_{2}\right)=e^{i \theta_{0}} e^{i G\left(\theta_{1}\right)} F_{p}\left(\theta_{1}, \theta_{2}\right)$ and note that $F\left(\varphi(t), \omega_{1} t, \omega_{2} t\right)=H\left(\omega_{0} t, \omega_{1} t, \omega_{2} t\right)$. Since $H: \mathbb{T}^{3} \rightarrow \mathbb{C}$ is continuous it follows by unique ergodicity that $p(t) / t \rightarrow$ $\int_{\mathbb{T}^{3}} H\left(\theta_{0}, \theta_{1}, \theta_{2}\right) d \theta_{0} d \theta_{1} d \theta_{2}$. This is simply the zero'th Fourier coefficient of $H$ which vanishes. Hence $p(t) / t \rightarrow 0$ and we have sublinear drift.

It remains to consider the case $n_{0} \omega_{0}+n_{1} \omega_{1}+n_{2} \omega_{2}=0$ where $n_{0} \geq 1, n_{1}, n_{2}$ are integers (written in lowest terms). Define the continuous function $J: \mathbb{T}^{2} \rightarrow \mathbb{C}$

$$
J\left(\theta_{1}, \theta_{2}\right)=e^{-i n_{1} \theta_{1}} e^{-i n_{2} \theta_{2}} e^{i G\left(n_{0} \theta_{1}\right)} F_{p}\left(n_{0} \theta_{1}, n_{0} \theta_{2}\right) .
$$

Then $F\left(\varphi(t), \omega_{1} t, \omega_{2} t\right)=J\left(\tilde{\omega}_{1} t, \tilde{\omega}_{2} t\right)$ where $\tilde{\omega}_{1}=\omega_{1} / n_{0}, \tilde{\omega}_{2}=\omega_{2} / n_{0}$. Since $\tilde{\omega}_{1} / \tilde{\omega}_{2}$ is irrational, it follows from unique ergodicity on $\mathbb{T}^{2}$ that $p(t) / t \rightarrow \int_{\mathbb{T}^{2}} J\left(\theta_{1}, \theta_{2}\right) d \theta_{1} \theta_{2}$. We obtain linear drift if and only if the zero'th Fourier coefficient of $J$ is nonzero; otherwise the drift is sublinear. It follows from condition (6.4) that $e^{i G} F_{p}$ consists only of Fourier modes of the form $e^{i\left(a+k_{1} \ell\right) \theta_{1}} e^{i k_{2} \theta_{2}}, k_{1}, k_{2} \in \mathbb{Z}$. Hence $J$ has Fourier modes of the form $e^{i\left(n_{0}\left(a+k_{1} \ell\right)-n_{1}\right) \theta_{1}} e^{i\left(n_{0} k_{2}-n_{2}\right) \theta_{2}}$, and the result follows.

Proposition 6.6 Suppose that the underlying $\mathbf{S E}(2)$-equivariant vector field is $C^{5}$. For almost every $\omega_{0}, \omega_{1}, \omega_{2} \in \mathbb{R}$, the drift on the relative torus is bounded.

Proof By Herman's Theorem, we may suppose that the changes of coordinates in Proposition 6.4 are $C^{5}$ so that $F_{\varphi}$ and $F_{p}$ are $C^{5}$ in equations (6.3). The remainder of the proof is similar to the one in [20] (but requires less differentiability for $F_{\varphi}$ due to the dependence only on $\theta_{1}$ ). As in the proof of Proposition 6.5, we can write

$$
p(t)=\int_{0}^{t} e^{i \omega_{0} s} e^{i G\left(\omega_{1} s\right)} F_{p}\left(\omega_{1} s, \omega_{2} s\right) d s
$$

where $e^{i G} F_{p}: \mathbb{T}^{2} \rightarrow \mathbb{C}$ is $C^{5}$. The resulting decay of the Fourier coefficients of $e^{i G} F_{p}$ together with the appropriate Diophantine condition on the frequencies $\omega_{0}, \omega_{1}, \omega_{2}$ guarantees as in [20] that $p(t)$ can be expressed as a uniformly convergent series.

Remark 6.7 In the context of forced symmetry breaking, LeBlanc [19] proves results on linear drift/boundedness in relation to phaselocking/irrational torus flows similar to Propositions 6.2, 6.3 and 6.6. The analogue to Proposition 6.5 (which relies on the equivariant Denjoy theorem, Corollary 2.4) is not discussed in [19].

Acknowledgements This research was supported in part by an EPSRC Advanced Research Fellowship (JL), by a Leverhulme Research Fellowship (IM), and by the Nuffield Foundation $(\mathrm{CW})$. 


\section{References}

[1] V. I. Arnold. Small denominators, 1: Mappings of the circumference onto itself. Amer. Math. Soc. Transl. 46 (1965) 213-288.

[2] V. I. Arnold. Geometrical Methods in the Theory of Ordinary Differential Equations. Grundlehren 250, Springer, New York, 1988.

[3] D. K. Arrowsmith and C. M. Place. An Introduction to Dynamical Systems. Cambridge Univ. Press, Cambridge, 1990.

[4] P. Ashwin and I. Melbourne. Noncompact drift for relative equilibria and relative periodic orbits. Nonlinearity 10 (1997) 595-616.

[5] H. W. Broer, M. Golubitsky, and G. Vegter. The geometry of resonance tongues: a singularity theory approach. Nonlinearity 16 (2003) 1511-1538.

[6] P. Chossat and M. Golubitsky. Iterates of maps with symmetry. SIAM J. Math. Anal. 19 (1988) 1259-1270.

[7] B. Fiedler. Global Bifurcation of Periodic Solutions with Symmetry. Lecture Notes in Math. 1309, Springer, Berlin, 1988.

[8] M. J. Field. Symmetry breaking for equivariant maps. Algebraic Groups and Related Subjects (G. Lehrer et al., ed.), Australian Math. Soc. Lecture Series, Cambridge Univ. Press, 1997, pp. 219-253.

[9] M. J. Field and R. W. Richardson. Symmetry breaking and branching patterns in equivariant bifurcation theory, I. Arch. Rational Mech. Anal. 118 (1992) 297-348.

[10] M. Golubitsky, J. E. Marsden and D. G. Schaeffer. Bifurcation problems with hidden symmetries. Partial Differential Equations and Dynamical Systems (W. E. Fitzgibbon III, ed.), Pitman Research Notes in Math., San Francisco, 1984, pp. 181-210.

[11] M. Golubitsky and I. N. Stewart. Hopf bifurcation in the presence of symmetry. Arch. Rational Mech. Anal. 87 (1985) 107-165.

[12] M. Golubitsky, I. N. Stewart, and D. G. Schaeffer. Singularities and Groups in Bifurcation Theory, Vol. II. Appl. Math. Sci. 69, Springer, New York, 1988.

[13] J. Guckenheimer and P. Holmes. Nonlinear Oscillations, Dynamical Systems, and Bifurcations of Vector Fields. Appl. Math. Sci. 42, Springer, New York, Heidelberg, Berlin, 1990.

[14] M.R. Herman. Mesure de Lebesgue et nombre de rotation. Geometry and Topology (J. Palis and M. de Carmo, eds.), Lecture Notes in Math. 597, Springer, New York, 1977, pp. 271-293. 
[15] R. Krikorian. Global density of reducible quasi-periodic cocycles on $\mathbf{T}^{1} \times \mathrm{SU}(2)$. Ann. of Math. 154 (2001) 269-326.

[16] M. Krupa and M. Roberts. Symmetry breaking and symmetry locking in equivariant circle maps. Physica D 57 (1992) 417-435.

[17] J. S. W. Lamb and I. Melbourne. Bifurcation from discrete rotating waves. Arch. Rational Mech. Anal. 149 (1999) 229-270.

[18] J. S. W. Lamb, I. Melbourne, and C. Wulff. Bifurcation from periodic solutions with spatiotemporal symmetry, including mode interactions and resonances. J. Diff. Eq. 191 (2003) 377-407.

[19] V. G. LeBlanc. Rotational symmetry breaking for spiral waves. Nonlinearity 15 (2002) $1179-1203$

[20] M. Nicol, I. Melbourne, and P. Ashwin. Euclidean extensions of dynamical systems. Nonlinearity 14 (2001) 275-300.

[21] D. Ruelle. Bifurcations in the presence of a symmetry group. Arch. Rational Mech. Anal. 51 (1973) 136-152.

[22] B. Sandstede and A. Scheel. Super-spiral structures of meandering and drifting spiral waves. Phys. Rev. Lett. 86 (2001) 171-174.

[23] B. Sandstede, A. Scheel, and C. Wulff. Bifurcations and dynamics of spiral waves. J. Nonlinear Sci. 9 (1999) 439-478.

[24] C. Wulff, J. S. W. Lamb, and I. Melbourne. Bifurcation from relative periodic solutions. Ergod. Th. Es Dynam. Sys. 21 (2001) 605-635. 